\title{
Development of miniaturized, spectroscopically assisted Penning gauges for fractional helium and hydrogen neutral pressure measurements ${ }^{\mathrm{a}}$
}

\author{
K. Flesch ${ }^{1, \mathrm{~b}}$ and T. Kremeyer ${ }^{1}$, O. Schmitz ${ }^{1}$, V. Soukhanovskii ${ }^{3}$, U. Wenzel ${ }^{2}$ \\ ${ }^{1}$ University of Wisconsin - Madison, Department of Engineering Physics, Madison, WI 53706, USA \\ ${ }^{2}$ Max Planck Inst. for Plasma Physics, 17491 Greifswald, Germany \\ ${ }^{3}$ Lawrence Livermore National Laboratory, Livermore, CA, USA
}

(Presented XXXXX; received XXXXX; accepted XXXXX; published online XXXXX)

Direct measurements of the helium $(\mathrm{He})$ fractional neutral pressure in the neutral gas around fusion devices is challenging because of the small mass difference between the abundant $\mathrm{D}_{2}$ molecules and the He ash which will be produced by D-T fusion. To study He exhaust, an in-situ Penning gauge system is being developed at UW-Madison that is optimized for good pressure and high spectroscopic sensitivity. Three different anode geometries have been studied regarding their vacuum electrostatic fields, light output, and ion current. The light output of the two new anode configurations are at least one order of magnitude above currently available designs, hence improving the spectroscopic sensitivity at similar total neutral pressure resolution.

\section{INTRODUCTION}

In fusion reactors, deuterium-tritium (D-T) plasmas will be heated to ignition and release large amounts of energy. The D-T fusion reaction has a by-product of helium ash, which if not exhausted efficiently enough, can begin to dilute the D-T plasma. To study He exhaust in current plasma devices, vacuum gauges utilizing the Penning trap principle ${ }^{1}$ have been used for measurement of total neutral pressure assisted by spectroscopy ${ }^{2}$ to resolve $\mathrm{D}$ and He. This approach was shown to be a very versatile method for partial pressure measurement of neutral He and molecular $\mathrm{D}_{2}$, which is challenging because they have almost identical masses. It has been used on plasma devices including TEXTOR, JET and DIII-D ${ }^{2,3,4}$. The measurements on these devices have all been done with large commercial Penning gauges, the Alcatel CF2P, that use permanent magnets to sustain the Penning discharge. This has been shown to be a reliable approach but is limited in time resolution by moderate light output. In addition, these gauges can only be mounted on the outer periphery of the device, so the vacuum time scale defines the measurement quality and interpretation. It is necessary, therefore, to develop a vacuum gauge using the Penning trap principle which can be inserted deep into the vacuum volume for instance directly into the divertor housing - in order to obtain full in-situ measurements. To accomplish this, the in-situ gauge must be small enough to be mounted near the divertor and edge regions, which will necessitate it use the inherent external field. The National Spherical Tokamak Experiment-Upgrade (NSTX$\mathrm{U})$ already has a custom gauge that can make fast total neutral pressure measurements located at various points around the torus [5], which was the starting point for this study. At the same time, we aim on preparing an in-situ fractional neutral pressure measurement inside the island divertor housing of the recently started stellarator Wendelstein 7-X (W7-X) to study the properties of the island divertor.

\section{INITIAL TEST AT W7-X}

For W7-X, a commercial Penning gauge, Alcatel CF2P, has been installed on an outboard vacuum flange in the device midplane in order to conduct a generic feasibility test. Helium emission lines were measured down to a base pressure of $10^{-5}$

\footnotetext{
${ }^{a}$ Contributed paper published as part of the Proceedings of the 21st Topical Conference on High-Temperature Plasma Diagnostics in Madison, Wisconsin, USA.

b)Author can be contacted at: kbflesch@wisc.edu
}

mbar and $\mathrm{H}_{\alpha}$ emission lines down to $10^{-6}$ mbar with an integration time of 10s. Successful measurement of the total as well as the fractional neutral pressures of $\mathrm{He}$ and $\mathrm{H}$ was demonstrated with the system at W7-X. The Thorlabs CS175 compact CCD spectrometer that was used features a dispersion range of 500 to $1000 \mathrm{~nm}$ with FWHM accuracy $<0.6 \mathrm{~nm}$ at 633 $\mathrm{nm}$ and a possible integration time between $10 \mu \mathrm{s}$ to $60 \mathrm{~s}$. The initial test has shown that the time resolution needs to be improved.

\section{NSTX-U PENNING GAUGE}

Currently several custom-built Penning gauges are installed on NSTX- $U^{5}$. The design of these gauges is depicted in Fig. 1. It was modified from the Penning gauges used on the Princeton Divertor Experiment (PDX) $)^{6}$ and has the unique ability to use either a magnetic field from permanent magnets mounted on the cathode plates or the external field generated from the field coils of NSTX-U itself. This allows for the gauge to be located either farther out on the pumping ducts or very close to the divertor and edge regions of the tokamak while still giving accurate neutral pressure measurements. With a bias voltage of $3.12 \mathrm{kV}$, this gauge can operate in a limited range from $1.3 \times 10^{-4}-2 \times 10^{-3}$ mbar. However, this gauge has not yet been used for partial pressure measurement.

\section{VACUUM TEST STAND}

To test different anode designs, an ultra-high vacuum test stand has been set up at UW-Madison. The attainable final pressure of the system is $<3 \times 10^{-9} \mathrm{mbar}$ with a turbopump volume flow rate of $1050 \mathrm{l} / \mathrm{s}$ for $\mathrm{H}$ and $1320 \mathrm{l} / \mathrm{s}$ for He. The test stand is equipped with a $400 \mathrm{~W}$ high voltage power supply ranging from $0-5000 \mathrm{~V}$, a Varian FRG-700 gauge measuring pressure from

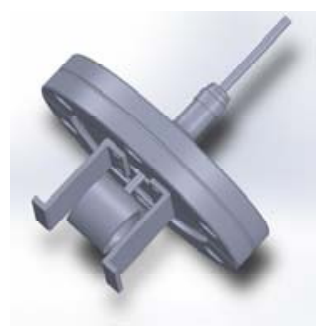

FIG.1. CAD drawing of the modified-PDX gauge in use on NSTX-U 
$1 \times 10^{-9}$ mbar up to atmosphere, and a gas inlet system which can supply $\mathrm{He}$ and $\mathrm{H}$. The optimized candidate anodes were tested with a magnetic field of $70 \mathrm{mT}$ from a pair of permanent magnets.

\section{ANODE DESIGNS}

Three anode designs were tested and are shown conceptually in the left column of Fig. 2 with an actual photograph of the discharge obtained on the right side. Since the optimized gauge must be able to use the external fields of the devices which it is mounted on, the starting point for the optimization process was the modified-PDX gauge design on NSTX-U. This was built and tested on the vacuum test. A discharge was achieved with this design from $1500-2750 \mathrm{~V}$ at a pressure of $5.0 \times 10^{-5} \mathrm{mbar}$ but at higher voltage did not ignite a discharge until above $1.0 \times 10^{-2}$ mbar. This anode design has achieved the desired sensitivity to total pressure, but as can be seen in Fig. 2.a, it has very low light output, which limits the time resolution of the spectral analysis.

Based on this, two new anodes were designed aiming for better optical access to the Penning discharge and enhanced discharge light altogether. The original NSTX-U configuration was changed to more open designs that allowed for viewing into the cylindrical portion of the gauge where the electrons are trapped and the neutrals are ionized. The first of the new designs attempted to look into middle of the cylinder by taking out a cut and leaving two thin rings $1 / 8$ " wide located the same distance away from plates as the cylinder. The schematic for this is in Fig. 2.b with a picture of the discharge. The second design was a thin ring $1 / 8$ " wide in the middle in order to see into the discharge from the sides. The geometry of this design was very similar to the Alcatel CF2P gauge. The schematic for this anode is in Fig. 2.c also with a picture from the discharge. All pictures in Fig. 2 were taken with comparable camera exposure and aperture settings and hence the light emissivity can be compared between the three designs. This visual inspection shows that the two new anode designs are far superior in terms of light emission. The next step is to qualify stable discharge conditions for the ion current measurement which represents the neutral density in the gauge volume. A monotonic increase of the ion current with pressure in the reference chamber must be found to enable calibration and eventual extraction of absolute neutral pressure values.
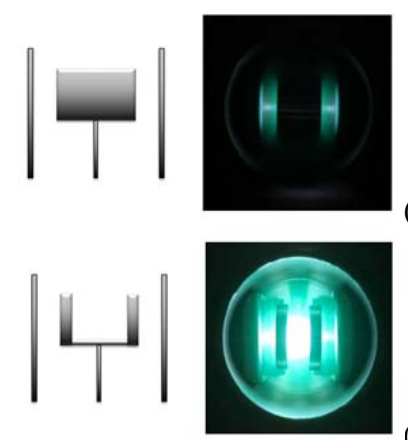

(a)
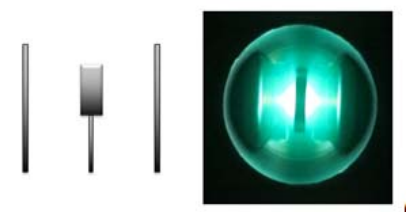

(c)

FIG. 2. Anode design schematics (not to scale) and pictures taken with a $20 \mathrm{MP}$ camera of the (a) NSTX-U anode, (b) double ring anode, and (c) single ring anode. Magnetic field is horizontal for this view.

\section{ANODE CURRENT}

Pressure sweeps in both directions were taken for all three anodes and the ion current at the cathodes was measured. The $\mathrm{I}\left(\mathrm{p}_{\mathrm{n}}\right)$ measurements are shown in Fig. 3. The tube anode generates about a factor of two stronger ion current compared to the ring and double ring anodes. In all three anodes, there are voltages in which the measured current does not increase monotonically with pressure. This could be due to the plasma switching to different discharge regimes or points at which instabilities form ${ }^{7,8}$. The most significant one was seen with the tube anode at $1500 \mathrm{~V}$ in the high $10^{-3}$ mbar pressure range. Despite this, it was still possible to find at least one operating voltage where current increased monotonically through the entire pressure sweep and could be feasible for total neutral pressure measurements. Because of the steep ion current curve, the tube anode seems to be the most sensitive design.

\section{LIGHT INTENSITY}

To quantitatively compare the light output of the three anodes, the CCD spectrometer was used. The brightness of four helium lines was compared at the same pressure and integration time. The comparison of the light intensities is shown in Fig. 4. The ring anode as well as the double ring anode are an order of magnitude brighter than the NSTX-U Penning gauge. The double ring anode creates the brightest discharge which can be, depending on the operating anode voltage, about 2.5 times brighter than the discharge of the ring anode. Note that the light output of the NSTX-U gauge is so small, it is not visible on the graph.

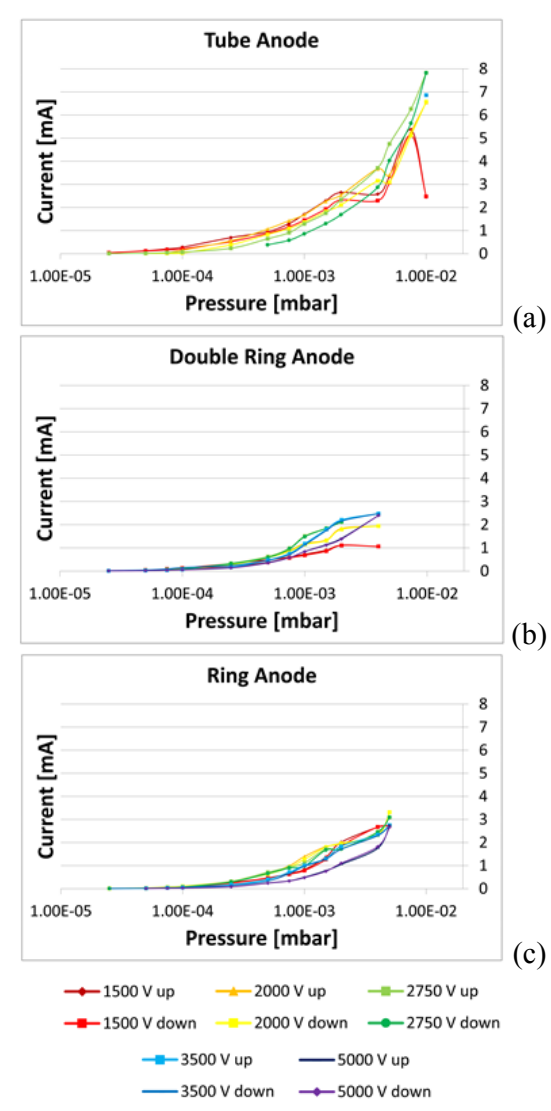

FIG. 3. Measured ion current at the cathode for the (a) NSTX-U anode, (b) double ring anode, and (c) single ring anode at various voltages while sweeping the pressure up and down. 


\section{VACUUM ELECTRIC FIELD MODELS}

A simple model of the vacuum electrostatic field for each anode was computed in order to add insight into the experimental results and aid in further optimization. The modelling was done in MATLAB using the finite difference method, and the electric field maps are shown in Fig 5 for all three cases. The model assumes axisymmetry, and the electric field is analyzed in one horizontal plane at the mid-plane of the gauge. Electrons become trapped in the areas where the electric field lines are perpendicular to the magnetic field, which is vertical in that view, due to ExB drifts. The ions, which are much more massive, practically follow the field lines directly to the cathode plates at the top and bottom. It can be seen that the only areas where the electric and magnetic fields are perpendicular for the tube (Fig. 5.a) and single ring (Fig 5.c) anodes are directly inside the anode and at the plane across the exact middle. The electric field strength for the ring anode, though, is much weaker than the tube anode, which leads to the ions moving slower towards the plates and the possibility for more collisions as well as recombination events. This could potentially be the reason for the large increase of light intensity from this anode. The double ring anode shows the interesting feature of having large regions between the rings with perpendicular electric and magnetic fields. This increases the volume where electrons are trapped, which further increases the chances for ionization events. The electric field strength in this center region is weaker than the tube anode, which again means slower ions and the chance for more recombination events and additional enhancement of light emission. This analysis suggests that the new anode designs increase the trapping volume and at the same time reduce the electrical field strength. This can increase the ion dwell time in the trap and enhance the interactions with the neutral gas, which increases the light emission level.

\section{SUMMARY}

Two different anode designs have been developed that have at least an order of magnitude brighter discharge than the presently available Penning gauges. The existing NSTX-U gauge served as reference, and it was seen that this gauge is the most sensitive to neutral pressure. However the ion current sensitivities of the ring and double ring anode seem feasible for accurate total pressure measurement. Based on these tests and the models, a new anode will be designed as a double ring anode with wider

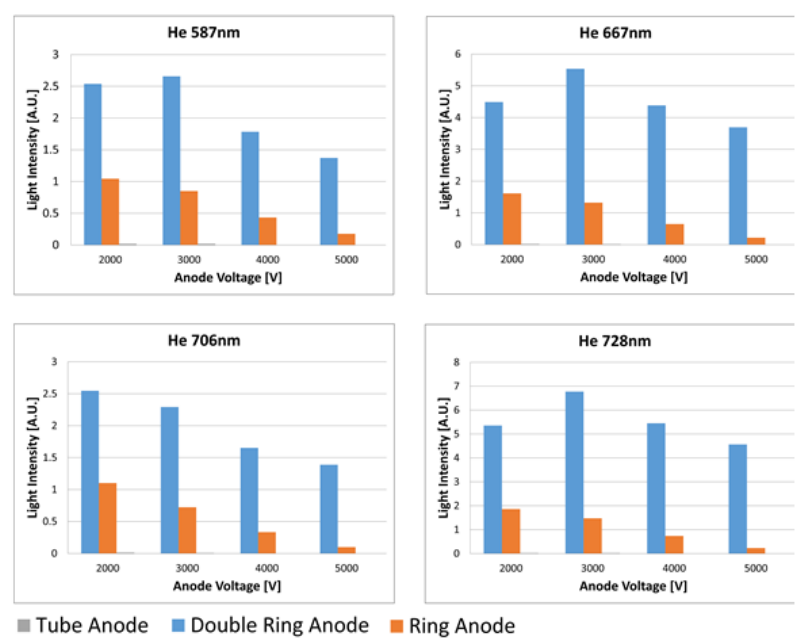

FIG. 4. Spectral line intensities for specified He emission lines for the three anode designs at various voltages

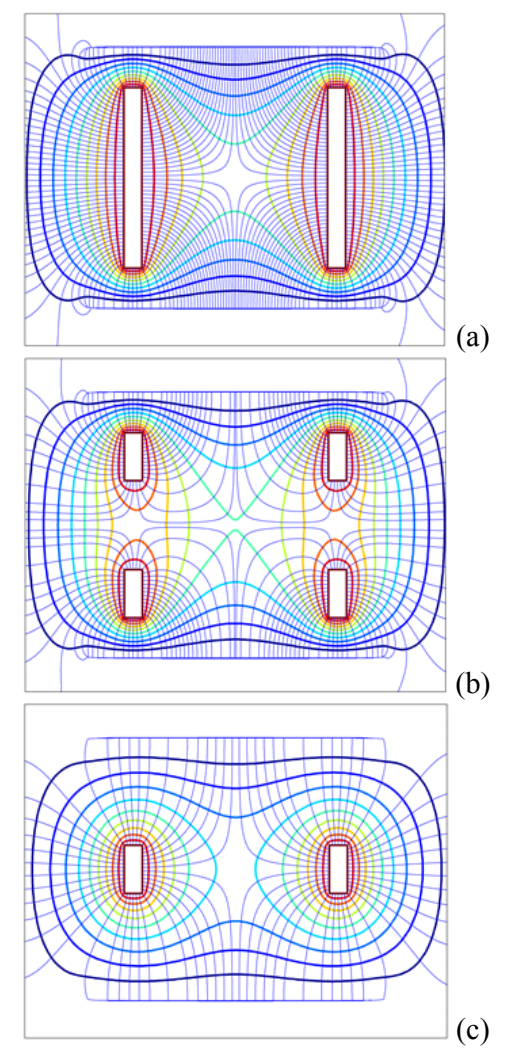

FIG. 5. Vacuum electrostatic field lines and equipotential surfaces for the (a) cylindrical anode, (b) double ring anode, and (c) single ring anode at $2000 \mathrm{~V}$. Magnetic field is vertical for this view

rings and a thinner gap. This shall maintain the good optical properties of the new anode designs but at the same time make the Penning trap plasma more resilient against the development of plasma modes and instabilities, which prevent a monotonic calibration of current with pressure. Future work also includes testing in different magnetic field strengths, especially above the transition field strength for developing these modes, as well as sensitivity to alignment with the magnetic field.

\section{ACKNOWLEDGMENTS}

The authors would like to thank the entire W7-X and NSTX$\mathrm{U}$ teams. This work was funded in part by the Department of Energy under grants DE-SC0012315 and DE-SC0014210 and DE-AC52-07NA27344. This work has been carried out within the framework of the EUROfusion Consortium and has received funding from the Euratom research and training programme 2014-2018 under grant agreement No 633053. The views and opinions expressed herein do not necessarily reflect those of the European Commission.

${ }^{1}$ F.M. Penning, Physica III, no. 9 (1936).

${ }^{2}$ T. Denner, K. H. Finken and G. Mank. Rev. Sci. Instr. 67, 3515 (1996)

${ }^{3}$ D. L. Hillis, C. C. Klepper, M. Von Hellermann, J. Ehrenberg, K. H. Finken, G. Mank. Fus. Eng. Des. 34-35 (1997) 347-351

${ }^{4}$ M. Wade et al. Phys. Rev. Lett. 74, 2702 (1995).

${ }^{5}$ R. Raman, H. W. Kugel, T. Provost, R. Gernhardt, T. R. Jarboe, and M. G. Bell. Rev. Sci. Instr 74, 1900 (2003).

${ }^{6}$ H. F. Dylla. Jour. Vacu. Sci \& Tech. 20, 119 (1982).

${ }^{7}$ P.A. Redhead, Vacuum 38, 8-10 (1988)

${ }^{8}$ C.C Klepper, R.C. Hazelton, F. Barakat, M.D. Keitz, and J.P. Verboncoeur. Int. Jour. of Plas. Sci. \& Eng. 2008 (2008) 